Die Preussische Militair - Pharmakopöe. Handbuch für Aerzte und Apotheker zum Gebrauch im Frieden und im Kriege. Bearbeitet von Carl Wach, Apotheker. Berlin 1854.

Dieses Werk ist dem Ober-Stabs-Apotheker K leist gewidmet. Die Einleitung spricht den Wunsch aus, dass alle neuen deutschen LandesPharmakopöen sämmtlich in Ein deutsches Dispensatorium vereinigt würden, mit Herstellung von gleichem Maasse und gleichem Gewichte. Da dieses sobald nicht zu erwarten, meint der Verf., so würde es leichter thunlich sein, eine deutsche Militair-Parmakopöe einzuführen, da die deutschen Heere vermöge ihrer Bundes-Organisątion nur als ein geschlossenes Ganze zu betrachten seien. Auch dieser Wunsch wird leider sobald nicht erfüllt werden, da auch in Militair-Angelegenheiten am Bunde nicht immer Einigkeit herrscht, die allein zur Einheit führen kann. Die Zweckmässigkeit solcher Einigung leuchtet natürlich jedem Verständigen ohne Weiteres ein. Der Verf. stellt dann heraus, dass sich die Preussische Militair-Pharmakopöe vollkommen bewährt habe, in gesundheitsrücksichtlicher Hinsicht für das Heer günstig gesorgt werde, der Kosten-Anschlag allerdings in Betracht komme, die Qualität der Arzneien alle Bürgschaft für Güte und Heilkraft gäbe. Wenn der Verf. S. VII ausspricht, dass den bewährten einheimischen Mitteln in der Militair - Pharmakopöe ihr Platz gesichert sei, so ist das in der Ordnung. Wenn aber die Rede davon ist, dass der oft beschwerliche transatlantische Import (warum nicht: „Einführung vom Auslande“?) leicht durch Blokade, Continentalsperre gehemmt werden könne, so ist diese Furcht wohl übertrieben, da wir seit $1810-1816$ dergleichen nicht $z a$ fürchten gehabt haben und für die Folge immer weniger zu fürchten sein wird, da einzelne Schifffahrt treibende Nationen immer neutral sein und so die Mittel für Arzneibereitung nicht fehlen werden. Leitender Grundsatz sollte nur der sein, im Arzneischatze jederzeit das wirksamste Mittel dem weniger wirksamen vorzuziehen: denn je wirksamer das Mittel ist, um so kürzer die Dauer der Krankheit. Dass nicht überall die vorbandenen Mittel in bester Qualität angeschafft sind, sondern bisweiten dem wohlfeilen der Vorzug gegeben, davon fehlt es nicht an Beweisen, wofür auch dieses Archiv Belege liefert. Daran kann natürlich nichts Anderes die Schuld haben, als die nicht immer ausgewählten sichern Quellen des Bezuges und die manchmal fehlende Prüfung in einzelnen Arzneidepots.

Der Abschnitt I. handelt vom pharmaceutischen Personal der Armee. Das dienstthuende Personal für Feld - Apotheken in einem Armee-Corps beläuft sich auf 11 Personen. Die Pflichten sind kurz erwähnt.

Der Abschnitt II. handelt vom Arzneibedarf des Heeres, den Militair - Apotheken der Lazarethe. Darin heisst es unter andern: Die contractlichen Lieferungen der Medicinalwaaren, wie sie zur Zeit noch mit den Civil - Apothekern bestehen, dürften auf diese Weise sehr bald aufhören. Mir scheint dieser Ausspruch sehr unzweckmässig und die Maassregel, wenn sie zur Ausfübrung käme, nicht ganz gerecht gegen die Apotheker.

S. 19 unter den Requisiten des Laboratoriums ist auch die Rede von Colirtïichern, hier wären wohl die zweckmässigen Colirtrichter von Porcellan, Zinn, gut verzinntem Blech an ihrer Stelle.

Das Mitschleppen von Windöfen, Destillirblasen, Extractkesseln, 
Kapellenöfen, Pressen, Schmelztiegeln, Schneidebrett, Wiegemesser ist doch wohl im Felde zu beschwerlich und durch Beziehung der nöthigen Mittel aus den nächsten Apotheken für den Fall des Bedarfs leichter zu decken.

\section{Die Receptirkunst.}

Die hier gegebenen Vorschriften sind im Allgemeinen durchaus zweckgemäss.

Nach dieser Einleitung ist die Militair-Pharmakopöe selbst abgedruckt, so dass auf der rechten Seite der lateinische Text, auf der. linken die Uebersetzung in deutscher Sprache erscheint.

Die Pharmacopoea selbst entbält in der ersten Abtheilung die einfachen und die zusammengesetzten Arzneimittel, mit sammt den Gaben, in denen sie verordnet werden, als kleine, mittlere und grösseste.

In der zweiten Abtheilung die zubereiteten und zusammengesetzten Mittel der Preussischen Landes-Pharmakopöe.

Die dritte Abtheilung umfasst die präparirten und zusammengesetzten Mittel der Militair - Pharmakopöe, die bekannt sind und hier natürlich unverändert wiedergegeben werden mussten. In einem Nachtrage sind erwähnt: Aq. phagedaenica nigra, Collodium, Tinctura Nucum Vomicarum Rademacheri.

Hiernach folgt: Anweisung zur Prüfung und Aufbewahrung der Arzneimittel.

\section{A. Chemischer Theil.}

Bei Emplastr. Cantharid. konnte unter den Prüfungsmitteln auch der Geruch zu Hülfe genommen werden.

Wenn es bei Emplastr. adhaesivum heisst: ,je älter, desto besser", so möge das doeh nur bedingungsweise gelten dürfen.

\section{B. Pharmakognostischer Theil.}

Colophonium. - Wer sonst das Colophon und sein Herkommen nicht kennt, wird es nach der Beschreibung $\mathbf{S} .160$ schwerlich kennen lernen. Es geht nicht einmal hervor, zu welcher Art von Körpern es gehört.

Folia Cardui benedicti. - Weshalb die Cardobenedictenblätter schmierig genannt werden, ist mir nicht klar.

Folia Digitalis. - Nicht allein im Harz, sondern auch in Thüringen häufig vorkommend.

Rad. Jalapae. - Zu den Verfälschungen ist auch die mit getrockneten, in Jalapentinctur getauchten Früchten zu zählen, die durch den verstorbenen Dr. Ingenohl aufgefunden und von deren Vorkommen ich mich überzeugt habe.

Rad. Rhei. - Zu den wirksamen Bestandtheilen der Rhabarber dürften doch wohl das Phäoretin, Erytroretin, Grysophansäure gehören.

Rad. Salep. - Fine neue Verfälschung hat man in kleinen Wurzelknollen von Colchicum erkannt, die treilich gar leicht zu entdecken sein wird.

Gedachtes kleines Werkchen erscheint seinem Zwecke vollkommen angemessen und darf somit allen Militair-Apothekern und Aerzten empfohlen werden.

Dr. L. F. Bley. 


\section{H. Gerste Preistabellen.}

Unter den Titel: „Arznei- und Preistabellen", für die Receptur zur leichten, sicheren und besonders schnellen Berechnung der Arzneipreise, nach den Preisen der Königl. Preuss. Arzneitaxe ansgearbeitet, hat $\mathbf{H}$. Gerste ein Schriftchen herausgegeben, welches mit vollem Rechte empfohlen werden kann, wo in den Apotheken die Königl. Preuss. Arzneitaxe Geltung hat.

Diese nur so geringen Raum einnehmenden 'Tabellen kürzen die oft so zeitraubende Arbeit des 'Taxirens sehr ab, und gewähren daher dem im 'Taxiren weniger Geiibten, eben so wie auch dem darin Geübteren grossen Vortheil, indem er sicher ist, dass er in der richtigen Taxirung der Recepte dem Publicum sowohl, als dem Principal gerecht wird und vor Fellern bewahrt, zu welchen das Berechnen im Kopfe häufig führen kann. Da Alles vom Gran bis zur Unze berechnet ist, konnten Brüche vermieden werden. Vor den Strichen in den Quadraten stehen die Silbergrosehen, nach den Strichen die Pfennige. Der besseren Uebersicht halber wechseln doppelte und einfache Colonnenstriche ab. $\mathrm{Da}$, wo sich die betreffenden wagerechten und senkrechten Colonmen schneiden, steht die gewünschte Zahl, z. B. die Drachme kostet 6 Sgr. 8 Pf., was 15 Gran? so findet man das Resultat, wenn man die wagerechte Colonne, in welcher der Drachmenpreis von 6 Sgr. 8 Pf. steht, verfolgt, ebenso die senkrechten, in der 15 Gran stehen, sie treffen sich bei 1 Sgr. 8 Pf., was der Preis für 15 Gran wirklich ist. Als Taxminimum sind 3 Pf. angenommen. Die erste Tabelle enthält die Berechnung einer Unze in 7 Drachmen von 1 Sgr., die Unze bis zu 10 Sgr. 10 Pf. Die zweite Tabelle enthält die Berechnung einer Drachme in Scrupel und Gran, von 1 Sgr. bis zu 7 Sgr. 2 Pf. die Drachme. Die dritte Tabelle endlich enthält die Berechnung des Scrupels von 1-19 Gran von $2 \mathrm{Sgr}$. if Pf. bis $10 \mathrm{Sgr}$.

Vorzüglich in sehr freqnenten Geschäften der grösseren Städte, wo gewöhnlich die meisten Recepte sogleich bezahlt werden, wird dem Receptarius dadurch eine grosse Erleichterung verschafft werden. Ich habe mich von der Nützlichkeit dieser von $H$. Gerste herausgegebenen Tabcllen vollkommen überzeugt, und kann daher meinen Herren Collegen die Anschaffung derselben für ihre Apotheken zu ihrem eigenen Nutzen empfehlen.

Dr. L. F. Bley. 